\title{
ALA Library Bill of Rights interpretations
}

\author{
By Patricia Senn Breivik
}

\section{Access to electronic information, services, and networks}

ast spring the ACRL Board was presented with a copy of a recently developed interpretation of the Library Bill of Rights on access to electronic information, services, and networks for endorsement. After reading the document, several Board members had concerns about it, which they raised when the item came before the Board for discussion and action.

The Board drafted a response to the interpretation expressing its discomfort with some of its phrasing. It asked the Intellectual Freedom Committee to defer taking action on the interpretation at the Annual Meeting and to consider its comments, but the decision was made by Judith Krug, director of ALA's Office for Intellectual Freedom, to proceed in taking it before Council for a vote. At Council the Board discovered that there were several ALA units and many individuals who shared ACRL's con- cerns. When the vote came to the floor of Council it was defeated.

Since the Annual Conference, ACRL sent letters to the other ALA divisions expressing its concern about the relationship between the ALA Intellectual Freedom Committee and divisions, and to the Intellectual Freedom Committee highlighting its concerns. ACRL is working with representatives from other divisions to address specific wording changes in the interpretation, and divisional leaders have agreed to discuss relations between divisions and the Intellectual Freedom Committee at Midwinter. At press time, I talked to Candace Morgan, chair of ALA's Intellectual Freedom Committee, who assured me that ACRL's input was important and that a new version of the interpretation would be issued shortly.

The interpretation, ACRL's response to it, and letters to the Intellectual Freedom Committee and to other ALA divisions are being shared with you for your information. Please feel free to contact any of the members of the ACRL Board of Directors to answer questions you may have or give comments on these documents.

\section{Access to Electronic Information, Services, and Networks: An Interpretation of the Library Bill of Rights (version 1.4, 6/25/95)}

[a new version is expected shortly]

The world is in the midst of an electronic communications revolution. Based on its constitutional, ethical, and historical heritage, American librarianship is uniquely positioned to address the broad range of information issues being raised in this revolution. In particular, librarians address intellectual freedom from a strong ethical base and an abiding commitment to the preservation of the individual's rights.

Freedom of expression is an inalienable human right and the foundation for self-gov- ernment. Freedom of expression encompasses the right to freedom of speech and the corollary right to receive information. Such rights extend to minors as well as adults. Libraries and librarians facilitate the exercise of these rights by providing access to, identifying, retrieving, organizing, providing instruction in the use of, and preserving recorded expression regardless of format or technology.

The American Library Association expresses the basic principles of librarianship in its Code of Professional Ethics and in the Library Bill of 
Rights and its Interpretations. These serve to guide professional librarians and library governing bodies in addressing issues of intellectual freedom and the rights of the people they serve.

Issues arising from the still-developing technology of computer-mediated information need to be approached and regularly reviewed from a context of constitutional principles and estahlished policy so that fundamental and traditional tenets of librarianship are not swept away.

It is the nature of electronic information that it flows freely across boundaries and barriers despite attempts by individuals, governments, and private entities to channel or control it. Even so, many people, for reasons of technology, infrastructure, or economic status do not have access to electronic information.

In making decisions on how to offer access to electronic information, each library should consider its mission, goals, objectives, and cooperative agreements, and the needs of all the people it serves. Such considerations will actdress the rights of users, the equity of access, and information resources and access issues.

\section{The rights of users}

All library system and network policies, procedures or regulations relating to electronic resources and services should be scrutinized for potential violation of user rights.

User policies should be developed according to the policies and guidelines established by the American Library Association, including Guidelines for the Development and Implementation of Policies, Regulations and Procedures Affecting Access to Library Materials, Services and Facilities.

Users have the right to be free of interference and unreasonable limitations or conditions set by libraries, librarians, system administrators, vendors, network service providers, or others. This specifically includes contracts, agreements, and licenses entered into by libraries on behalf of their users.

No user should be restricted or denied access for expressing or receiving constitutionally protected speech. No user's access should be changed without due process, including, but not limited to, notice and a means of appeal.

Although electronic systems may include distinct property rights and security concerns, such elements may not be employed as a subterfuge to deny users' access to information. Users have a right to information. training and assistance necessary to operate hardware and software provided by the library.

Users have the right of confidentiality in all of their activities with electronic resources and services provided by the library, and the library shall ensure that this conficlentiality is maintained. The library also should support, by policy, procedure, and practice, the user's right to privacy; however, users should be advised that because security is technically difficult to achieve, electronic communications and files could become public.

The rights of users who are minors shall in no way be abridged. ${ }^{1}$

\section{Equity of Access}

Electronic information, services, and networks provided directly or indirectly by the library should be readily, equally, and equitably accessible to all library users. Once the decision is made to provide access to electronic information, the user must not be required to pay to obtain the information or use the service. When resources are insufficient to meet demand, rationing service may be necessary to provide equitable access. All library policies should be scrutinized in light of Economic Barriers to Information Access: an Interpretation of the Library Bill of Rights and Guidelines for the Development and Implementation of Policies, Regulations and Procedures Affecting Access to Library Materials, Services and Facilities.

\section{Information Resources and Access Issues}

Electronic resources provide unprecedented opportunities to expand the scope of information available to users. Libraries and librarians should provide material and information presenting all points of view. This pertains to electronic resources, no less than it does to the more traditional sources of information in $\mathrm{l}$ braries. ${ }^{2}$

Libraries and librarians should not deny or limit access to information available via electronic resources because of its allegedly controversial content or because of the librarian's personal beliefs or fear of confrontation. Information retrieved or utilized electronically should be considered constitutionally protected unless determined otherwise by a court with appropriate jurisdiction.

Providing access to electronic information, services, and networks is not the same as selecting and purchasing material for a library collection. Libraries may discover that some 
information accessed electronically may not meet a library's selection or collection development policy. It is, therefore, left to each user to determine what is appropriate. Parents who are concerned about their children's use of electronic resources should provide guidance to their own children.

Just as libraries do not endorse the viewpoints or vouch for the accuracy or authenticity of traditional materials in the collection, they do not do so for electronic information.

Libraries must support access to information on all subjects that serve the needs or interests of all users regardless of the user's age or the content of material. Libraries and librarians should not deny access to information on the grounds that it is perceived to be frivolous or lacking value.

Libraries have a particular obligation to pro- vide access to government publications available only in electronic format.

In order to prevent the loss of information, libraries may need to expand their selection or collection development policies to ensure preservation, in appropriate formats, of information obtained electronically.

\section{Notes}

1. See Free Access to Libraries for Minors: An Interpretation of the Library Bill of Rights; Access to Resources and Services in the School Library Media Program; and Access for Cbildren and Young People to Videotapes and Other Nonprint Formats.

2. See Diversity in Collection Development: An Interpretation of the Library Bill of Rights.

$E d$. note: Contact the ALA Office for Intellectual Freedom for these documents.

\section{Memo to ALA Intellectual Freedom Committee from ACRL Board of Directors}

The Board of ACRL, ALA's largest division, has substantive reservations about the Library Bill of Rights for Electronic Information Services and Networks and asks that this document not be finalized until its concerns are addressed and resolved. These are two overriding general concerns

A.) Documents such as this one do not address the realities of academic life and, therefore, can put academic librarians in untenable positions. For example, to say that "Libraries and librarians should not limit access to information on the grounds that it is perceived to be frivolous or lacking value" is to assume that planned collection development has no relevance to academic libraries and that librarians have unlimited funds for acquisitions. Moreover, since faculty members are often most eager to have libraries buy materials which are of almost exclusive interest to them, statements such as these seem to imply that academic librarians are morally obligated to respond no matter how esoteric the request to campus curriculum and research priorities.

B.) Adding another interpretation to the Bill of Rights appears to expand the complexity of the issue by proliferating the documents relevant to intellectual freedom. If an ever increasing number of interpretations are needed then perhaps the basic document needs to be changed.
In addition, it is particularly noteworthy that the text points to keeping the electronic access consistent with library policies toward print materials. It is important not to let the "rapidity of delivery" which the technology provides blind us to the essential intellectual freedom issues that have traditionally been of concern to librarians and which they have defended vigorously.

Simultaneously there are some difference because of the nature of computers in an academic and research library setting which need to be examined further. These include:

(I) Page 2, Equity of access. It is not clear whether "rationing service" includes charging for services beyond some basic level of activity. Therefore, for example, if online searching of a commercial vendor's database is an established service, can the library charge for service beyond some maximum allowable amount to which all have access? Unless rationing includes charging for use beyond some basic level then I fear that many institutions would not support the statement and their policies would conflict with it. Another aspect of the rationing has occurred to me and that is differential charging (or even prohibition) for services depending on the purpose of the use. Thus for example, online search charges might be billed to a patron who is seeking personal information as opposed to information in support of a 
course assignment. Many institutions use this as a form of rationing.

(II) Page 3, Information Resources, 5th paragraph. This statement on access to all materials is inconsistent with the widely applied policy in academic settings of restricting use of computers for games and other nonacademic uses. This policy varies among institutions in its strictness, but at a minimum, states that class-related use of computers has priority and those using computers for non-class purposes must give way to those who need it for class purposes. More stringent limits include no game playing at all on the theory that wear and tear on equipment, especially on mouse and keyboard, shortens the life of the machine so that the institution cannot replace the equipment as frequently as would be necessary. In academic libraries there generally is a restriction that limits some computer use and access to students and faculty of the institution. It is important to make distinctions between such things as CD-ROMs and public online systems and use of computer resources such as central system information, E-mail and other uses that require authorization and an individual computer account.

(III) Page 3, Information Resources and Access Issues, third paragraph. The distinction between access and ownership is too casually made here. The library has a responsibility to apply collection development criteria to the materials for which it provides access. However that selection should not be driven by issues of personal taste and should not be censorious. The two sentences that conclude the paragraph apply equally well to collections the library owns as well as to information for which it provides access. Bottom line, we can endorse the last two sentences but I don't think they are exclusively applicable to electronic information because it is accessed rather than owned.

It is also incorrect to say, as the first sentence implies, that libraries only provide access to electronic information and do not select and purchase such material. The purchase of CDs and multimedia programs falls under a collection development policy as do print resources.

The Board received version 1.4 dated June 25, 1995, of these interpretations of the Library Bill of Rights which it has not had the opportunity to study at this conference. Should a newer version address some of our concerns we thank you, but further discussion is cleariy needed once units have had the time to study the document. No formal approval of the document should be given until such discussion can take place.

\section{Memo to ALA Division Presidents and Executive Directors from Patricia Senn Breivik}

It was not with pleasure that ACRL, through its ALA Councilor, raised concerns about the document "Access to Electronic Information, Services, and Networks: An Interpretation of the Library Bill of Rights" at the last meeting of the ALA Council in Chicago. Yet, when an informal request to Judith Krug, Director, ALA Office for Intellectual Freedom, to slow the process down to allow for addressing concerns in a positive fashion rather than on the floor of Council was rebuffed, we felt we had no choice. Very shortly thereafter we learned of another division that had very similar concerns, and the discussion at Council showed that the concerns were more broadly felt than by just two divisions.

Now, some months later, I am forwarding to you the attached memo which the ACRL Executive Committee reviewed at its recent meeting and which we are forwarding to Candace Morgan, Intellectual Freedom Com- mittee (IFC) Chair, and Judith Krug. We wanted to share it with you so as to encourage other ALA divisions to take time also-whether or not the particular issues raised from an academic perspective are of concern to your membership - to respond substantively to the Library Bill of Rights, interpretation for "Access to Electronic Information, Services, and Networks" prior to the Midwinter Meeting and also to copy other division presidents so that we may all be aware of the collective concerns that exist.

In addition to the issue of the wording of the electronic interpretation, there is also a process issue of concern to us, i.e., the relationship of the Intellectual Freedom Office and Committee to the divisions and other major units of ALA. As mentioned earlier, when ACRL leadership requested a delay in bringing forward the draft of the electronic interpretation to Council, its request was summarily dismissed be- 
cause, we were told (as was restated in Krug's American Libraries September 1995 interview), IFC was "being harassed to get this policy on the books" and therefore, the IFC was knowingly rushing the document through "faster than we have ever taken any intellectual freedom policies forward."

The question must be asked as to where the IFC gets such mandates: from ALA and its divisions and other major units, some unnamed and undefined group of individuals, and/or only IFC members and staff? Since, as of October 6, 1995, the ACRL Board has still not had any response to the memo it forwarded to IFC during the annual conference raising specific questions about draft 1.4 (see attached), it could be interpreted that, in practice, its sending requests for feedback from various ALA units is only perfunctory
I sincerely hope that is not the case, for there are already people outside librarianship who question the collective value of the individual cases championed by the IFC. Surely those people and the world in general should not see a situation where the well-motivated ef forts of the IFC are so rushed or so insensitive to divisional concerns and/or the operational realities of today's libraries as to force internal division within the ranks of intellectual freedom supporters.

ACRL is interested to know if your division also has any process concerns regarding response to its input into intellectual freedom documents. Therefore I am asking you to discuss the issue with your Executive Committee and as appropriate with your Board as background to a discussion at the Presidents' breakfast at Midwinter

\section{Memo to ALA Intellectual Freedom Committee from Patricia Senn Breivik}

It was not with pleasure that ACRL, through its ALA Councilor, raised concerns about the document "Access to Electronic Information, Services, and Networks: An Interpretation of the Library Bill of Rigbts" at the last meeting of the ALA Council in Chicago. In fact, given the very high esteem that academic institutions place on academic freedom, issues of intellectual freedom are doubly reinforced in the life of academic librarians. But, in our respect for the importance of academic and intellectual freedom, also comes a responsibility to speak up when specific actions and/or issues are questionable.

Let me highlight three general areas of concern with the current Intellectual Freedom activities before addressing the specific concerns of the electronics interpretation.

1.) It would seem that the very strength of the Intellectual Freedom effort (i.e., the high level of commitment of ALA members) may become its weakness, as each aspect of every possible infringement is explored and prescribed in great detail. The proposed "Access to Electronic Information, Services, and Networks" will be the sixteenth interpretation of the Library Bill of Rights. Just as the laws and prophets of the Old Testament were interpreted into literally thousands of minor regulations which, for all practical purposes, put compliance beyond all but a very small group of people, there reaches a point when increasing supplementary detail can have a negative impact on the overall effectiveness of the original intent. When this happens, the need to be able to put forward the principle of intellectual freedom in clarion call to others, as well as to serve as a reasonable conscience to daily practice is lost. We cannot allow continuing addition of complex interpretations of the Library Bill of Rights to have diminishing positive effects. The ACRL Board of Directors, therefore, urges the Intellectual Freedom Committee (IFC) to ag gressively explore how to simplify the interpretations to provide a more succinct and more effective support document(s) for the Library Bill of Rights.

2.) The ACRL Board would further point out the importance of guarding against wording in its documents that confuse nonlibrarians as to the juncture point between normal collection development efforts--which by their nature must involve purchasing some things and not purchasing others. Any statement that infers that libraries must or should buy or provide access to all materials wanted by its users is unrealistic in many settings. Comments in the electronic interpretation, for example, that state "once the decision is made to provide access to electronic information, the user must not be required to buy ..." or that "librarians should not deny (Bill cont. on page 40) 


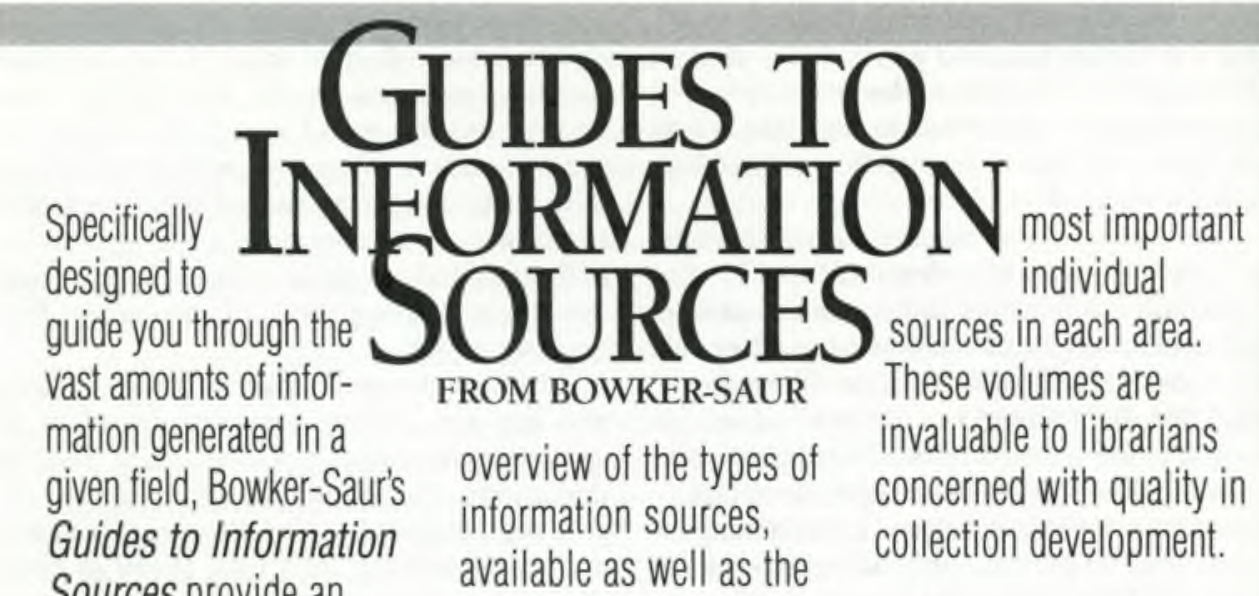

\section{NEW!}

Information Sources in

Architecture

2nd Edition

Edited by Valerie Nurcombe

1995 • 1-855739-094-6 • c . 500 pp • • 5900T

NEW!

Information Sources in

Engineering

3rd Edition

Edited by Ken Mildren and

Peler Hicks

1995 • 1-85739--057-1 • c. 600 po • \$100.00

NEW!

Information Sources in the

Life Sciences

4th Edition

Edited by H.V. Wyatt

$1995 \cdot 1-85739-070-9 \cdot c .250 \mathrm{pp} \cdot \boldsymbol{8} 775.00$

\section{Information Sources in}

Environmental Protection

Edited by Selwyn Eagle and

Judith Deschamps

1995 • 1.85739-062-8 • 300 pp. • \$70T

\section{Information Sources in}

Grey Literature

3rd Edition

By C.P. Auger

$1994 \cdot 1-85739-007.5$ • 170 pp $\bullet$ \$ $\$ 65.00$
Inlormation Sources in Physics

3rd Edition

Edited by Dennis Shaw

1994 • 1-85539012-1 • 475 pp. • $\$ 105.00$

Information Sources in

Chemistry

4th Edition

Edited by R.T. Bottle and

J.FB. Rowland

$1993 \cdot 1-85739-016-4$ • 350 pD $\bullet \$ 75.00$

\section{Information Sources in the}

Medical Sciences

4th Edition

Edited by Leslie Morton and

Shane Godbolt

1992 • 0-86291-596-1 • $624 \mathrm{pp} . \$ \$ 100.00$

Information Sources in Patents

Edited by Peter Auger

$1992 \cdot 0-86291-906-1 \cdot 187 \mathrm{pp} \cdot \bullet 75.00$

\section{Intormation Sources in}

Sport and Leisure

Edited by Michele Shocbridge

$1992 \cdot 0-86291-901-0 \bullet 345 \mathrm{pp} \cdot \$ 955.00$

Information Sources for the

Press and Broadcast Media

Edited by Selwyn Eagle

$1991 \bullet 0.86291-900-2 \cdot 218 \mathrm{pp} \cdot \mathbf{\$} 70.00$
Inlormation Sources in Information Technology

Edited by David Haynes

$1991 \cdot 0-408-03285.5 \cdot 528$ pp $\bullet \$ 80.00$

COMING IN 1996

Information Sources in

Finance and Banking

Edited by R.G. Lester

March 1996 • 1-85739-037-7 • C. 550 pp • $\$ 950$. O

\section{Information Sources in Law}

2nd Edition

Edited by E.M. Logan and

J.R. Winterton

1996 • 1-85739-041-5 • c. 500 pp. $\bullet \$ 95.00$

Information Sources in Music

Edited by Lewis Foreman

$1996 \bullet 1-85739-075-X \cdot$ c. 400 pp. $\bullet \$ 80.00$

To order, or for more information,call Bowker-Saur toll-free at

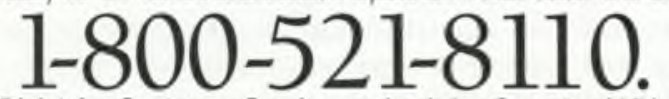

Dial 1 for Customer Service, and ask for Operator IA51.

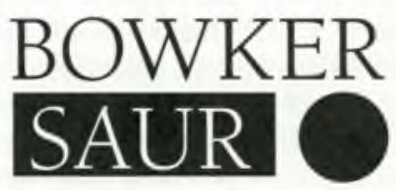




\title{
College \& Research Libraries News
}

\author{
INDEX TO VOL. 56 (1995)
}

\section{Compiled by Kathy L. (Kit) Dusky}

\author{
Edited by Eldon w. Tamblyn \\ Portland State University, Oregon
}

\section{A}

Abston, Deborah, "Racial \& ethnic diversity: information exchange," 31, 276, 716-17

Ahstracts solicited, 380

"ALADN: a new network for fundraising," Smith, 329

Academic or Research Librarian of the Year Award, 1995, 259 \& photo. 564; 1996, 563

"Academic reference service over e-mail: an update, "Bristow 459-62

"Accreditation assistance available," ACRL, 620-21

Accreditation: opportunities for library leadership," Bangert, 697-99

Acquisitions (by author, subject, or title): Alger, Horatio, 351; ALI, 200; Americana, 425; Amnesty Intl. USA, 40; Ancient Near Eastern seals (Porada), 579; Architecture (Clark, 578; Feiss, 501; Jacolss, 724; urban, 783 [\& photo., 784]); Ardis Publishers (Russ. lit. ), 40, 108; Band conducting (Hindsley), 725; Beckett, Samuel \& photo., 199; Broadfoor, Barry S (Canadian hist.), 108; Brown, Marcia J. (children's lit.) 501; Browning, James (music), 724-25; Bulgarian publications, 500-501; Burns, Ken, 40; Camus, Albert, 578-79 Canadian Coalition on Acid Rain, 351; Canadian hist. (Broadfoot), 108; Canadiana (Gibson), 200; Casey, Robert P. (politics), 784; Central Airlines, 200; Children's lit., 40 (Brown, 501; Cotsen, 199-200); Chinese lit., 199; Clark, Adèle Goodman (Women's Suffrage Movement), 725; Clark, Alson (architecture), 578; Clement, Arthur J. H., Jr. 784; CWA, 724; Cotsen, Lloyd E. (children's lit.), 199-200; Darby (Dr. \& Mrs. William J.) (nutrition), 501; Davies, Thomas M., Jr. (Latin America, hist.), 425; Drama, criticism (Whitakker), 200; Economics (Harrod), 662; Edwardian novels, 425; Engl. lit., 425; Feiss, Carl (architecture), 501; Fisher, Mary Wager (journalism), 501; Fitzgerald, F. Scort, 579; Flax, Herman J. (Physiatry), 425; Florin Japanese

FILING is word-by-word (ALA, 1968)

ABBREVIATIONS: Standard abbreviations are used except in titles. Names of some organizations-ALA, ACRL, LC, etc.-are also ahbreviated and are alphabetized as if spelled out.

\section{SPECIAL ABRREVIATIONS:}

appt. appointment prof. profile

foundation

prog. program

port. portrait

SPECIAL USAGES: More than one reference per page is indicated in parentheses. Under the heading "Acquisitions (by author, subject, or title)" parentheses may enclose donors' names (for subjects) or subjects (for named collections).
American Citizens League Oral Hist. Proj., 501; Foley Tom, 501; Fowler, Jack (military hist.), 282; Fraser, Sylvia, 40; Gaughan, William J. (U. S. Steel), 351; Gershwin George, port. (Siqueiros), 282; Gibson, Robert C., 200; Ginsberg, Allen, 39-40; Greene, Graham, 281-82, 425: Harrod, Roy (economics), 662; Henson, Walter R. (natural hist.), 579; Hickman, John R. (sound recordings), 108 ; Hindsley, Mark (Band conducting), 725; Hogg, James, 662-63; Homosexuality (ONE Institute), 351; Horatio Alger Soc., 351; Intl. Brigade of the Spanish Civil War, 351 Ironside, H. A (correspondence), 282; Jacobs, Jane (architecture), 724; Japanese Americans, 501 (\& photo.); Japanese hist. (Maruzen Pub. Co.), 663; Journalism (Fisher 501; Kendall, 725); Kahle, Keith Hayes, 200; Kelley, George (Sci. fic), 282; Kendall, George Wilkins (journal ism), 725; Latin America, hist. (Davies), 425; Liebling, William, 40; Lowry, Lois, 40; Lowry, W. McNeil, 784; Luce, Arthur Aston (philosoplry), 724; Maps, 724 (photos., 685 725; Soviet Union, 108); Maruzen Pub. Co. (Japanese hist.), 663; Maury Family, 784; Medicine, 425; Middle East Map Coll., 724 (photos., 685, 725); Military hist. (Fow]er) 282; Moody Church (Chicago), 282; Music (Browning, 724-25; Neumann, 40; westem, 784); Nash Finch Co. 725; NCCUSL, 200; NI'A, 200; Natural hist. (Henson), 579; Neumann (Frederick) Coll. (music), 40; Nutrition (Darby), 501; ONE Institute (homosexuality), 351; Philosophy (Luce), 724; Phono-... See Sound recordings; Physiatry (Flax), 425; Poetry, American (Warren), 199; Politic: (Casey), 784; Porada, Edith (ancient Near Eastern seals) 579; Proffer, Ellendea (Russ. lit.), 40, 108; Russ. lit. (Ardis Publishers, 40, 108; Proffer, 40, 108); St. Kliment Ohridski U. Press, 500-501; Salishury, Harrison E. \& port, 425 Schneider, Alan, 199; Sci. fic. (Kelley), 282; Siqueiros, David Alfaro (Gershwin port.), 282; Sound recordings (Hickman), 108; Soviet Union (maps), 108; Speaker of the House (Foley), 501; Taiwan publications, 199; Theatrical agents (Wood \& Liebling), 40; Thoreau, Henry David, 282 Tsukamoto (Mary) Japanese American Collection, 501 (\& photo.); UCC, 200; U. S. Steel (Gaughan), 351; Urban, Joseph (architecture), 783 (\& photo., 784); WaldensianMethodist Coll ., 40; Warren, Robert Penn, 199; Whitakker. Herbert, 200; Williams, Tennessee, 663 (\& port.); Women's Suffrage Movement (Clark), 725; Wond, Audrey, 40

Acquisitions (by institution): American U., 108; Autry Mus. of Western Heritage, 784; Boston Coll., 199, 281-82, 500501, 724; Brandeis U., 351; Brit. Lib., 662; CSU, Sacramento, 501 (\& photo.); Coll. of Wm. \& Mary, 784 Columbia U., 425, 663; Duke U., 501; Emory U., 199 Huntington Lib., 578; McMaster U., 40; NYU, 425, 724; Northern Ill. U., 351; Notre Dame, 724; Ohio U., 199; Pa. State U., 784; Pierpont Morgan Lib., 579; Princeton U. 199-200; San Diego State U., 425; Stanford U., 39-40 
SUNY (Albany, 501; Buffalo, 724-25); United Theol. Seminary, 40; U. of Alberta, Edmonton, 108; U. of Buffalo, 282; UC, Irvine, 40, 108; U. of Colo., 40; U. of Fla., $578-$ 79 (Gainesville, 501); UI, U-C, 725, 784; U. of Manitoba, 108, 579; U of Md, Col. Park, 425; U of Minn., 40; UNC, CH, 40; U. of N. D., 725 (Grand Forks, 200); U. of Pa., 200; U. of Pittshurgh, 351; U. of Richmond, 40; U. of S. C., 579 , 662-63, 784; U. of Southern Calif, 351; UT (Arlingion, 200, 725; Austin, 40, 282, 425); U. of Toledo, 282; U. of Waterloo, 351; U. of Wis., Milwaukee, 724 (photos., 685, 725); Vanderbilt U. Med. Ctr., 501; Va. Commonwealth U., 425, 725; Washburn U., 282; Wash. State U., 501; Wheaton Coll., 282; York U., 200

"Active learning materials available via ftp, " ACRL, 687

Adalian, Paul T., Jr., "Cal Poly's multimedia approach to research," 10-16

Adam, Anthony J., "Internet resources for film and television," $397-400$

Adams, Ida G., deceased, 505

"Address the ACRL Board," 775

Africa seen by American Negroes, map (cover, no. 2)

"Africana file available on Internet," U. of Mich., 73

Alexander, Adrian, "Wedding doesn't stop conference attendance" \& photo., 533-34

Allen, Nancy, news note, 726

Almand, Jean, retired, 667

"Alternative Care Homepage," Bradsher, 779

Amato, Sara, "Internet reviews," 32-33, 102-3, 190-91, 277-78, $346-47,420-21,493-95,574-75,659-60,714-15,778-79$

AAHE, National Conference, 1995, "Teaching, leaming, and technology roundtable," 326, 344

American Book Prices Current, "There's still time to bid on two silent auction items," 708

ALA. Annual Conference, 1995, "ACRL in Chicago: forecasting the future" \& photos., 550-58; "ACRL preconferences in Chicago, "271-72; "ACRL programs and meetings," [AC-1]AC-16 (no. 5); "BCALA hosts fundraising gala," 311; "Meet the editors," 333; "Order your Annual Conference audiocassettes," 553

ALA, Annual Conference, 1996, "ALA bids Mickey Mouse adieu, 5-6; "Call for sci/tech abstracts," 380

ALA, "ACRL gift spurs donations to ALA's fund," 531

ALA, Executive Board, "ALA bids Mickey Mouse adieu," 5-6

ALA, "Fair use in the electronic age: serving the public interest. ${ }^{n}$ 1995, draft, 24, 46

ALA, IFC, "Comments on issues of access to information," 312-13

ALA, "Libraries sought for traveling exhibit," 752

ALA, Midwinter Meeting, 1995, 267-68; "What's hot and what's not, ${ }^{, 2} 236-39$

ALA, Midwinter Meeting, 1996, "There's still time to bid on two silent auction items, ${ }^{n} 708$

ALA, "Visit the homepage," 534

"ALA bids Mickey Mouse adieu," ALA, Executive Board, 5-6

"ALA gets own Internet server," 754

"ALA Handhook corrections," 752

"ALA holds information literacy hearing at Midwinter, ${ }^{\text {" }} 688$

"ALA Library Fellows set for overseas assignments" \& photo. 729

"ALA mobilizes library advocates" (logos \& poster), 78

"ALA offers library advocacy training," 6

"ALA reorganization destined for further study: actions at ALA Council, Chicago, 1995," Hisle, 533

"ALA under attack," 687

"Amerirech Library Services names new president," 232-33

Amory, Hugh, retired, 787

AmRhein, Richard, "Internet resources for music, ${ }^{n} 760-63$

"And the drawing winners are ...," 137 (photo., 135)

Anderson, Susan, "St. Petersburg Jr. College reorganizes for the '90s," 541-46

André, Pamela Q. J., appt., 352

"Applause for ACRL's support of ALA Goal 2000," Stoffle, 270

"Apply for the NLW grant," 76
"Applying for professional positions, "Larsen, 415-17

Appointments, 43-47, 109-12, 202-3, 283-85, 352-54, 427-29, $503-5,581-83,664-67,726-28,785-87$

Armstrong, Judith, retired, 112

Amold, JoAnn, appt, 664

"Art on Film moves to Columbia," Program for Art on Film, 140

ACRL, Academic or Research Librarian of the Year Award, $1995,259, \&$ photo. 564; 1996, 563

ACRL, "Accreditation assistance available," 620-21

ACRL, "And the winners are ...," 483-85

ACRL, "Annual conference programs and preconferences," 1995, 801

ACRL Annual report, 1993-1994 (55:653-72), clarification, $36 ; 1994-95,791-813$

ACRL, ANSS, "Interner resources for sociology," 639-43

ACRL, BIS, Bibliographic Instruction Publication of the Year Award, 1995, 262

ACRL, BIS, Innovation in Bibliographic Instruction Award, 1995, 264

ACRL, BIS, Miriam Dudley Bibliographic Instruction Librarian Award, $1995,258,260 ; 1996,563-64$

ACRL, BIS, "Offers active learning materials," 312

ACRL, BIS. See also ACRL, IS

ACRL, Board of Directors, "Address the ACRL Board," 775 "ACRL Board approves Marta Lange/CQ Award," 137; "ACRL, Choice contribute to ALA Goal 2000," 137; "ACRL to build Community Information Organizations," 313 . "Charts new direction," 571-72

ACRL, Board of Directors, Carla Stoffle open letter, 270

ACRL, Board of Directors, "Highlights" (Feb., 240, 257; June \& photos., 560-61)

ACRL, Board of Directors, 1994-95, photo., 560, photo. \& roster, 808; 1995-96, photos., 561

ACRL, Budget \& Finance Comm. Chair, "Financial report" \& port., 809-813

ACRL, "Candidates for AL.A Council," 269

ACRL, "Charting new directions," 791-813

ACRL, CLS, CLIP notes, $\# 17$ (55:61), 84; \#20, 74; \#21, 140

ACRL, CLS, Emergency planning and management in college libraries (CLIP note \#17) published (55:61), 84; Library services for non-affiliated patrons (CLIP note $\# 21$ ) published, 140; Managing student employees in college libraries (CLIP note \#20), published, 74; "Research agenda for college librarianship," 470-71, 485, comments, 645

ACRL, CICLS, Community College Learning Resources Achievement Awards, 1995, 264-65

ACRL, Doctoral Dissertation Fellowship, 1995, 260, \& photo. $569 ; 1996,566$

ACRL, EBSS, Distinguished Education and Rehavioral Sciences Librarian Award, 1995, 261; 1996, 565

ACRL, EBSS, "Seeks editor," 7; "Seeks editor and moderator," 312

ACRL, EALS, "English \& American Lit Section starts listserv," $379-80$

ACRL, Exec. Director, "Letter" \& port., 796-97

ACRL, ECLSS, "Seeks researchers," 381, 383

ACRL, "Hahn and Miller share plans for ACRL," 178-83

ACRL, Hugh C. Atkinson Memorial Award, 1995, 258; 1996 564

ACRL, IS, "Active learning materials available via ftp," 687; "Call for technostress reactor panelists, " 619; "Guidelines for instruction programs in academic libraries," 1995. draft, 767-69; "Revising guidelines," 532-33; "Wants ideas for new plan," 753

ACRL, IS, Innovation in Instruction Award, 1996, 565-66

ACRL, IS, Instruction Publication of the Year Award, 1996, 569

ACRL, IS. See also ACRL, BIS

ACRL, "Internet and proceedings topics of two new ACRL publications" \& photos., 453-54

ACRL, Internet resources: a subject guide \& photo., 453

ACRL, LPSS, Marta Lange/CQ Award, 137, 1996, 565

ACRL, "Meet the ACRL staff" \& ports., 648-50 
ACRL, "Meet the editors," 333

ACRL, "Memorial resolution honoring William Andrew Moffett (1933-1995), , 232

ACRL, Midwinter Meetings, 1995, 240, 257

ACRL, Midwinter Meetings, 1996, "ACRL discussion groups,

776; "ACRL meerings in San Anionio," 771-76

ACRL "More on information literacy data," 231, 382

ACRL, MLA, "New MLA listserv," 623

ACRL National Conference, 1995, "And the drawing winners are ..., 137 (photo., 135); "ACRL's silent auction offers great prizes," 172-73, 200; "Carnegie Museum in Pittsburgh" photo., 3; "City of three rivers" (photos., cover, no. 1 , no. 3); "Conference proceedings available," 531; "Did you save your postcards?" 5 ; "Dining in Pittsburgh: from elegant to jazzy," 167-69; "Have dinner with a local librarian," 169: "Internet room" photo., 391: "Pittshurgh a success!" \& photo., 311; "Pittsburgh: a walk around town" \& photo., 95-97; "Preliminary program, ${ }^{n}$ P-1-P-20 (no. 1); "Proceedings now available" \& photo., 752; "Register for ACRL by Feb. 28 and save," 71; "Silent auction" photo., 392, "What's new? An update from Pittsburgh" $\&$ photos., 385-92, correction, 548; "What's new, part two: more from Pittshurgh" \& photos. 473-79

ACRL, National Conference, 1997, "ACRL in Nashville: save these dates," 140; "Choosing our futures: ACRL's 8th National Conference," 394-95; "In Nashville: a focus on dialogue," 646; "Nashville Cub riverboat" photo., 377

ACRL, "New book banishes stereotypes," 73

ACRL, "New titles from ACRL in 1995," 807

ACRL, Pasi-President, photo., 477

ACRL, President, "Becoming beyond-library-walls librarians" \& port., 468-69, response, 636-38; "Meet the candidates," 772; "Message" \& por., 792-93

ACRL, RBMS, Karharine Kyes Leab and Daniel J. Leab American Book Prices Current Exhibition Catalogue Awards, 1995, 265 (photo., 229); 1996, 568-69

ACRL, Science and Engineering Conference proceedings: $a$ guide to sources for identification and verification \& photo, 453-54

ACRL, STS, "Call for sci/tech abstracts," 380; "Discussions at Midwinter," 8; "Seeks new logo," 234

ACRL, STS, Eunice Rockwell Oberly Award, 1995, 262

ACRL, "Search committees aid ACRL," 451, 453

ACRL, "Standards for college libraries," 1995, approved, 245

57; foundations, 330-31, 337

ACRL, SPC, "Strategic plan," final draft, 1995, 401-3, 412

ACRL, "Thanks for your support, ${ }^{\text {" } 467}$

ACRL, "The time to lead, " 270

ACRL "Top priorities," 798

ACRL, "Topper joins staff," 619, 622-23 (photo., 650)

ACRL, ULS, "Busy at ALA in Chicago," 534; "Distance education: a fad or the furure?" 243 ; "Guidelines for university undergraduate libraries," 1995, draft, 338-41; "Revision of the mission of a university undergraduate library: model statement," 339; "Undaunted by wintry Philadelphia," $242-43$

ACRL, "Update on information literacy data," 75, updates, 231, 382 ACRL, Vice-President, "Message" \& port, 794-95

ACRL, WESS, Martinus Nijhoff International West European

Specialist Study Grant, 1995, 261-62; 1996, 566-68

ACRL, "The year in review," 798-807

"ACRL award opportunities" \& photos., 563-69

"ACRL award winners, 1995," 800

"ACRL Board approves Marta Lange/CQ Award," 137

"ACRL candiclates for 1996 elections," 765-66, 769

"ACRL charts new direction," 571-72

"ACRL, Cboice contribute to ALA Goal 2000," ACRL, Board of Directors, 137

"ACRL colleagues," 813

"ACRL collecting information literacy data" \& survey form, 382

"ACRL collects information literacy data," 8 , updates 75,231 , 382
"ACRL conference proceedings available," 531

"ACRL discussion groups," 776

"ACRL electronic communications," 74-75, corrections, 234

"ACRL gift spurs donations to ALA's fund," 531

"ACRL honors the 1995 award winners," Spiegel, 258-65

"ACRL in Chicago: forecasting the future" \& photos., 550-58

"ACRL in Nashville: a focus on dialogue," veldof, 646

"ACRL in Nashville: save these dates," ACRL, National Conference, 1997, 140

"ACRL launches mentoring program," 687

"ACRL listserv corrections," 234

"ACRL meetings in San Antonio," 771-76

"ACRL official documents published in 1994-95," 803

"ACRL preconferences in Chicago," 271-72

"ACRL programs and meetings 1995 ALA Annual Conference," [AC-1]-AC-16 (no. 5)

"ACRL project update, ${ }^{\text {" Hale, } 454}$

"The ACRL publications program" \& photos., Werking, 332-33

"ACRL seeks nominees for office, ${ }^{n} 270$

"ACRL 7th National Conference proceedings now available" \& photo., 752

"ACRL staff," 797

"ACRL strategic plan, "final draft, 1995, 401-3, 412

"ACRL supports teleconferences for library assistants" \& photo., Hale, 452

"ACRL to build Community Information Organizations," 313

"ACRL to cosponsor online information access workshop," 311

"ACRL wants you!" 651-56

"ACRL's journals," 806

"ACRL's key partnering organizations," 804

"ACRL's mission," 795

"ACRL's silent auction offers great prizes," 172-73, 200

ARL, OMS/DORAL, "Library fundraising rips and resources," 328

ARL, "Transborder ILL guidelines available," 687

"Association of Research Libraries (ARL) Statistics," Morgan, 494-95

Atkins, Thomas V., retired, 667

Atkinson (Hugh C.) Memorial Award, 1995, 258; 1996, 564

"Atomic Bomb Casualty Commission" \& photo., 623

Austin, Gabriel, letter to the ed., 466

Awards, 137, 228-29, 258-65 (14), 426, 503, 581, 664, 800

Ayers, Janet, retired, 787

\section{B}

Baker, Doug, photo., 312

Baker \& Taylor, Academic or Research Librarian of the Year Award, 1995, 259, \& photo, 564; 1996, 563

Bakshis, Bob, photo., 452

Balconi, William, Brother, S.]., retired, 47

Baltics Online," Small, 190

Bangert, Stephanie Rogers, "Accreditation: opportunities for library leadership," 697-99

Barry, Carol J., appt, 503

Bartelstein, Andrea, photo., 264

Baruch Coll., "Wins awards" \& photo., 531-32

"Baruch wins awards" \& photo., 531-32

Basefsky, Stuart M., "Pooled endowments: a new funding idea," 405-7

Battenfeld, Robert L., "LABSTAT-Bureau of Labor Statistics data server," 191

"BCALA hosts fundraising gala," 311

"Becoming a leader on campus," Mech, 409-12

"Becoming beyond-library-walls librarians" \& port., Breivik, 468-69, response, 636-38

Bell, George H., "Oberly Award" \& port., 262

"Benefit book auction a success," U. of Vt., 5

Bethel, Kathleen E., photo., 729

Bibliographic Instruction Publication of the Year Award, 1995,262

Biggs-Williams, Ann, appt., 109 
Gaboury, John, news note, 352

Gallegos, Bee, "Internet resources for education," 153-57, correction, 320

Gene Autry Western Heritage Mus., National Forest Dude Ranch Vacations, directory (cover, no. 6)

George, Melvin R., "Thoughts on leadership: an exchange," 636-38

George, Susan C., deceased, 429; "Library disasters: are you prepared?" \& photo., 80-84

Gerboth (Walter) Award, 1995, 426

"Get connected!" CGRL NewsNet, 100, 276, 457, 711

Getchell, Charles M., Jr., appt., 785

"Getting the 1992 library data," NCES, 8, updates, 75, 231, 382

Getty Ant History Information Program (AHIP), "Getty pilot project explores use of digital images," 139

"Getty pilot project explores use of digital images," 139

Ghikas, Mary W., appt., 284

"Gigantic Sycamores at Owl Creek, Ohio," Harvey (photo., cover, no. 9)

Goodyear, Mary Lou, "Wedding doesn't stop conference attendance" \& photo., 533-34

Goostree, Jane, retired, 505

Gorman, Michael, "Candidates for ALA president" \& port. $267-68$

"Got books here: a poem," Kunde, 754

GPO, "Government Printing Office expands database access," 72; "Purdue links Web to federal documents," 451

"Government Printing Office expands database access," Kelly, 72

Goya, Francisco, "Le descañona," 617

Graf, Francine, cluties \& port., 648; photo., 551

Grants (by grantee): Albion Coll., 723, 783; Alvernia Coll., 198; AAS, 783; ALA, 531, 752; Amer. Theol. Lib. Assoc, 107 Brown U., 281; Calif. School of Professional Psychology, San Diego, 107; Calif. State Polytechnic U., 350, 578; CRL, 107, 198, 350, 424; Chatham Coll., 424; CUNY, 662; Clark U., 350; Columbia U., 578, 783 ( \& photo., 784); Concordia U., 39; Cornell U., 424, 500; CLR, 281; Eastern Va. Med. School, 500; Emory U., 281; Harvard Coll., 723; Hoover Institution, 73; Ind. School of Medicine, 107; Ind. U., 424, 723, 783; LaGuardia Community Coll., 662; Lib. of Va., 662; La. State U, 107; La. Universities Marine Consortium, 39; Newberry Lih., 752 ; Ohio State U., 107, 578; Radcliffe Col1., 198; RLG, 198; Rutgers U., 39, 281, 783; Smjthsonian Inst., 424; Southern Ill. Collegiate Common Market, 5 ; Spring Hill Coll, , 424; SUNY, Albany, 723; Tex. SL, 39, 500Tex. Tech U., 350; Trenton State Coll., 198; U. Ctr. in Ga., 198; U. of Ariz., 198; U of Calif., 578 (Berkeley, 281 [2]; Davis, 39; LA, 281; SD, 690 [\& photo], Santa Cruz, 350, 500); U. of Fla., Gainesville, 107-8; U. of Hartford, 198 ; U. of 1ll., 198-99; U. of Manitoba, 350; U. of Md., Coll. Park, 723; U. of Mich., 500; U. of Montana, 662; U. of Nev. (LV, 723; Reno, 662); UNC, CH, 199, 500; U. of N. D., 783-84; U. of Notre Dame, 424; U. of Okla., 39; U. of Pa., 199; U. of Redlands, 199; U. of S. C., 662; U. of S. Fla., 723-24; UT, Austin, 578; U. of the Arts in Phila., 424, 783; U. of Utah, 281 ; U. of Wash., 281; Valparaiso U., 662; Vanderbilt U., 724; Va. Commonwealth U., 39, 350, 725; Wayne State U. \& ports., 350-51; WGBH, 281 (photo., 282); Wright State U., 281 (photo., 282)

Grants (by grantor): AAEA \& ports., 350-51; ALA, 350; Andrews and McMeel F, 107; Arcadia F, 198; Areas of Natl. Need Proj, 723; Bryson (Vaughn \& Nancy), 500; Canada, 578; CSSHRC, 39; Central Jersey Regional Lih. Coop., 198; CLR, 198; Culpeper (Charles E.) F., 350, 424; Delmas (Gladys Krieble) F., 198-99 (2); Geisel, Audrey S., 690 ( 8 photo.); GTE, 723-24; Getty Grant Prog., 424; Greenfield (Albert M.) F., 424; Herzog (Carl J) F., 198; HEA, 198, 281 (2), 424; III., 5; Indianapolis F., 107; IGT, 723; Japan F., 198-99; Kellogg (W. K.) F., 531, 783; LSCA, 39; Lilly Endowment, 107, 662; La., 107; MacArthur (John D. \& Catherine T.) F.,
531; McGregor Fund, 723; MCI F., 723-24; Mellon (Andrew W.) F., 107-8 (2), 198, 281, 424, 500, 578 (3); Mellon (Richard King) F., 424; Moore (Frank \& Sidney), 199; Mortensen (Wm \& Alice) F. 198; NASA, 281; NEH, 39, 73, $107,198,281$ (2), 350 (3), 424 (2), 500, 662, 723, 752, 783 (2) (\& photo., 784); NHPRC, 424, 500, 662 (3), 723, 783; NLC, 350; NLM, 39, 350, 500; Natl. Network of Libs. of Med., 39, 724 (2); NSF, 107, 281, 783; N.Y., 723; OCLC, 783; Ohio Humanities Council, 281; Reynolds (Kate B.) Charitable Trust, 199; Schafer, Robert L. "Robin," 662; SSHRC (C), 39; Sullivan (Joseph D. \& Sandra), 500; Summerlee F., 39; Tarplee, Evelyn M., 723; Terra F. for the Arts., 783; U. S. Dept. of Defense, 281; USDE, 578, 783; U. S. Dept. of the Interior Minerals Management Service, 39; U. S. West F., 783-84; Universal Press Syndicate and Cos., 107; Whitney-Carnegie Award, 350

"Grants and acquisitions," Thompson, 39-40, 107-8, 198-200, $281-82,350-51,424-25,500-501,578-79,662-63,723-25$, $783-84$

Gratch, Bonnie, "Accreditation: opportunities for library leadership," 697-99

Gray, Dorothy, retired, 667

Griffin, Cheryl, deceased, 203

Griffin, Mary Ann, deceased \& port., 668

Grochmal, Helen M., "Selecting electronic journals," 632-33, 654

Gropp, Dorothy M., deceased, 668

"Guest editorial," Jenkins, 450

"Guidelines for instruction programs in academic libraries," ACRL, 1995, draft, 767-69

"Guidelines for university undergraduate libraries," ACRL 1995. draft, 338-41

Gyeszly, Suzanne D., news note, 581

H

Hafner, Arthur W., appt., 43

Hahn, Bessie K., "Plans for ACRL" \& port., 178-81

Hale, Noreen, appt. \& port., 284; "ACRL project update," 454

"ACRL supports teleconferences for library assistants" \& photo., 452 ; duties \& port, 648

Halporn, Barbara, "Wins Nijhoff Award" \& port, 261-62

Hamilton, Leo, retired, 583

Hardesty, Larry, appt. \& port. 665

Harloe, Bart, appt. \& port., 43-45

"Harvard and MIT sign reciprocal access agreements," 688

Harvard Law School, Leab Award \& photo., 265

Harvard U., Leab Award \& photo., 265

Harvey, George, "Gigantic Sycamores at Owl Creek, Ohio" (photo., cover, no. 9)

Hary, Francesca L. \& Nicoletta Mattioli, "Hosting an international librarian," 162-64

"Have dinner with a local librarian," 169

"HealthWeb developed by CIC members," 623

Hedberg Jane, "Preservation news," 28, 100, 188, 275, 345 $419,491,573,658,713$

Helbig, Jack, duties \& port., 649

"Helping students delve deeper into books," Fogarty, 7045

Henderson, Carol C., "Copyright and fair use: maintaining the balance," 24

Henderson, Kathryn Luther, news note, 426

Hendon, Julia A., "Internet resources for anthropology," 8790,113

Highfill, William C., news note, 109

Hindmarsh, Doug, deceased, 354

Hine, Lewis, "Truant selling Saturday Evening Post, St. Louis Mo., $1910^{\prime \prime}$ photo., 529

Hirshon, Arnold, appt. \& port., 785

Hisle, W. Lee, "ALA reorganization destined for further study: actions at ALA Council, Chicago, 1995," 533; "Community College Award" \& port., 264-65; photos., 560-61, 808

Hitchingham, Eileen, appt., 427 
Hoadley, Irene B., "Customer service? not really," 175-76 retired \& port., 285-86

Hodges, T. Mark, retired, 583

Hodgson, Janet, retired, 667

Hoffman, Elaine, "Federal Communications Commission Gopher," 346-47

Hoffman, Irene M., "Fundraising: a selected list of Internet resources," 692-93, 717; "Library fundraising tips and resources," 328; "Teaching, learning, and technology roundtable," 326, 344

Hogan, Sharon, news note, 581; photo., 479

Holtz, Virginia, news note, 43

Hoover Institution, "Russian state archives will be added to RLIN," 71, 73

Horio, Nina D. P., deceased, 354

Horn, Steve, "Overdue notices: cutting costs and saving time," 8

Horne, Doug, "EFF Web the Electronic Frontier Founda tion, " 659: "Voice of America gopher," 32-33

Horny, Karen, appt., 665

"Hosting an international librarian," Hary, 162-64

"Hosting future librarians" \& photo., Marion, 481-82

Houser, Florence, retired, 505

Houston Academy of Medicine-Texas Medical Center Lilorary,

"Atomic Bomb Casualty Commission" \& photo., 623

Hugh C. Atkinson Memorial Award, 1995, 258; 1996, 564

Hughes, Joy, "Thoughts on leadership: an exchange," 636-38

"The Human-Languages Page," Robare, 660

"Human rights gopher," Chasse, 574-75

Hunt, Judith Lin, photo., 729

Huntington Lib, Leab Special Award of Merit, 265

Hupp, Stephen L., "Internet resources for conservatism, "46466, correction, 548; "Vote Smart Web (Project Vote Smart), $714(\log 0,715)$

I

"Implications of commercial document delivery," Coons, 626 31, comment \& response, 780

"In the Neus," Davis, 4, 70, 136, 230, 310, 378, 530, 618, 686 751

Ind. U., Bloomington, "Academic reference service over email: an update, "459-62, "The Life of a Book," 689-90

Ind. U., Leab Award honorable mention, 265

ISLA, "Everything you ever wanted to know about L.A.," 379

ISM, "Univ, of Alberta outsources cataloging," 140

Innovation in Bibliographic Instruction Award, 1995, 264

Innovation in Instruction Award, 1996, 565-66

ISI, Doctoral Dissertation Fellowship, 1995, 260, \& photo. $569 ; 1996,566$

ISI, Samuel Lazerow Fellowship, 1995, 260-61; 1996, 568

Instruction Publication of the Year Award, 1996, 569

"Instruction Section revising guidelines," ACRL, 532-33

"Instruction Section wants ideas for new plan," ACRL, 753

"IBM launches digital library initiative" \& photo., 380-81

"IEEE Computer Society gopher," Engel, 102-3

"IFLA '95: where Europe meets Asia," Ford, 644-45

Intl. Seminar on Lib. Management, "Facing the challenge of democratization," 324-25

Internet, "Africana file available," 73; "ALA gets own Internet server," 754: "ACRL electronic communications," 74-75, corrections, 234; "ACRL launches mentoring program," 687; "ACRL listserv corrections," 234; "Copyright service on the Internet," 451; "English \& American Lit Section starts listserv," ACRL, 379-80; "Everything you ever wanted to know about L.A." U. of Southern Calif., 379, "French cave paintings online," 139-40; "Fundraising: a selected list of Internet resources," 692-93, 717; "Get connected! jobs and news now on the Internet," $100,276,457,711$ "LC unveils THOMAS," 98; "Listserv on collective bargaining started," 690; "New MLA listserv," 623; "Online resources for Internet trainers," 535-39, 572; "Purdue links Weh to federal documents," 451; "Readmore sponsors
Backserv," 381; "Rettig on Reference' debuts," 688-89, "Selection criteria for Internet resources," 92-93; "Smithsonian libraries available," 233; "Stanford creating digital Dewey for Internet," 231; "Time to put the Internet in perspective," 144-47; "Using the Internet in serials management," 148-50, 176; "Visit the ALA homepage," 534; "Who uses the Internet?" 680

"Internet and proceedings topics of two new ACRL publications" \& photos, $453-54$

"The Internet Movie Database," Emmons, 346

Internet resources: a subject guide \& photo, ACRL, 453

"Internet resources for anthropology," Cohen-Williams, 87 90,113

"Internet resources for conservatism," Hupp, 464-66, correc tion, 548

"Internet resources for education," Gallegos, 153-57, correc tion, 320

Internet resources for film and television," Adam, 397-400

"Internet resources for law," Jacox, 18-20

"Internet resources for music," AmRhein, 760-63

"Internet resources for sociology," McMillan, 639-43

"Internet resources for the earth sciences," Cobb, 319-21, 325

"Internet reviews," Amato, 32-33, 102-3, 190-91, 277-78, 346$47,420-21,493-95,574-75,659-60,714-15,778-79$

"Irvine Valley gets new building," 751

Irwin, Margaret A, news note, 43

J

Jackson, Arlyne, news note, 202

Jacobsen, Kristin, "Time to put the Intemet in perspective, $144-47$

Jacobson, Frances F., "BIS Publication of the Year Award" \& port., 262

Jacobson, Michael J., "BIS Publication of the Year Award," 262 Jacox, Corinne, "Internet resources for law," 18-20

Jakeman, Carolyn E., deceased, 584

James, Jennifer, photo., 385; port., 556

Jebb, Marcia, retired, 583

Jedrey, Micheline, photo., 555

Jenkins, Althea H., duties \& port., 649; "Guest editorial," 450 "Letter from the executive director" \& port., 796-97; photos. 560-61, 808

Jenkins, Paul O., "Working with faculty to build collections," 322

Johnson, Richard D., retired \& port., 286

Jones, P. Alston, Jr., appt. \& port., 110

Josey, E. J., news note, 664

"Journal of electronic publishing created," U. of Mich., 71

"June 2 deadline for summer classified ads," CGRL news, 322

\section{K}

K. G. Saur Award for Best College \& Research Libraries Article, 1995, 260; 1996, 569

Karpisek, Marian, photo, 729

Kascus, Marie, "Using the Internet in serials management, $148-50,176$

Kaser, David, news note, 581

Katharine Kyes Leab and Daniel J. Leab American Book Prices Current Exhibition Catalogue Awards, 1995, 265 (photo. 229); 1996, 568-69

Kathman, Mike, photo., 476

Kaufman, Diane, "Building preservation awareness" \& posters, $707-8$

Kelly, Marie Caitlin, "Government Printing Office expands database access," 72; "Student retention and academic libraries" \& photo., 757-59

Kem, Carol Ritzen, "Internet resources for sociology," 639-43

Kennedy, Scott E., appt., 727

"Kent State builds new math library" \& photo., 71

Kirk, Thomas G., photos. 560,808

Klatt, Mel, retired, 112

Klein, Rhona, "Web Day in Portland, Oregon," 688 
Knecht, Michael, appt., 727

"Kright Riclder to acquire CARL Corp.," 531

Koenig, Melissa, photo., 759

Komara, Edward, Walter Gerhoth Award, 426

Kratz, Charles E., "Internet resources for education," 153-57, correction, 320

Kruus, Alar, retired, 787

Kunde, Brian, "Got hooks here: a poem," 754

\section{i}

"LABSTAT - Buredu of Labor Statistics data server," Battenfeld, 191

LaCroix, Michael, appt., 352

Land, Miss Roy, deceased, 286

Landesman, Berty, "CDnow! the Internet music store," 102

Lange (Marta)/CQ Award, 137; 1996, 565

Lanning, Roland, deceased, 203

Larsen, Lotte, "Paris Jages: a collection of everything regarding the City of light" \& logo, 659-60

Larsen, Suzanne T., "Applying for professional positions," 415-17

Latzke, Henry, news note, 426-27

Lazerow (Samuel) Fellowship, 1995, 260-61; 1996, 568

Leab (Katharine Kyes and Daniel J.) American Book Prices Current Exhibition Catalogue Awards, 1995, 265 (photo., 229); $1996,568-69$

Lee, Charles E., news note, 283

Lee, Sul, news note, 664

Lee, Tamera P., "NetVet" \& logo, 420-21

Lehman, Doug, news note, 109

Lener, Edward F., "Internet resources for the earth sciences," $319-21,325$

Lesnik, Pauline Tina, deceased, 113

Letters, 270, 466, 645, 780

"The librarian is in," Blewett, 701-3

"Libraries sought for traveling exhibit," ALA, 752

"Library disasters: are you prepared?" George, \& photo., 8084

"Library fundraising tips and resources," ARL, Hoffman, 328

"LC unveils THOMAS," 98

Library services for non-affiliated patrons (CLIP note \#21) published, ACRL, 140

"The Life of a Book, ${ }^{\prime \prime}$ 689-90

Lindsay, Lorin, appt. \& port., 727

"Listserv on collective bargaining started," 690

Longstreet, Christine, deceased, 608

Longstreet, Stephen, "Waiting," drawing (cover, no. 5)

"LSU book hazaar earns $\$ 58,000,{ }^{n} 751$

Lowry, Lina M., retired, 667

Lowry, Marcia Duncan, "Dining in Pittsburgh: from elegant to jazzy," 167-69; "Pittsburgh: a walk around town" \& photo., 95-97

Loyola U., "The librarian is in," 701-3

Luck, DeAnne, "Scholarly Societies Project," 493-94

Lucker, Jay K., retired, 505

Lumumba, Malikah Dada, photo., 729

Lynch, Mary Jo, "Coming in '96: IPEDS adds new data items," 622; "New national numbers on academic libraries" $(55$ : $630-31$ ), data available, 8 , updates, $75,231,382$

\section{$M$}

McArthur, Fran, retired, 47

McChesney, John, photo., 387

McConkey, Joan S., "Applying for professional positions," 415-17

McCord, S. Joe, news note, 503

McDonald, Peter, "Implications of commercial document delivery, ${ }^{n} 626-31$, comment $\&$ response, 780

McKinzie, Steve, "Research across the curriculum," 414, 417 , comment, 645

McMillan, Gary A., "Internet resources for sociology," 639-43

McNiff, Philip J., deceased, 354

McPhee, James S., deceased, 354
"Madonna in the hallowed halls," Metter, 710-11

Magpintay, J. Andrew, app , 352

Malone, Cheryl Knott, "United Nations gopher, ${ }^{\text {" }} 277$

Malone, Debbie, photo, 135

Maloy, Frances, photos, 560-61, 808

Managing student employees in college libraries (CLIP note \#20), published, ACRL, 74

Marcum, Deanna B., appt, 45

Marion, Yvonne N., "Hosting future librarians" \& photo., 481-82

"Marketplace challenges for research libraries," Byrd, 694-95

Marshall, John David, news note, 109

Marta Lange/CQ Award, 137; 1996, 565

Martin, Marilyn J., appt., 665

Martin, Robent S., appt. \& port., 352-53

Martin, Susan K., "Message from the president" \& port., 792 93; photos., $477,560-61,564,569,808$

Martinus Nijhoff International West European Specialist Study Grant, 1995, 261-62; 1996, 566-68

Martz, Frederick M., appt., 284

MIT, "Harvard and MIT sign reciprocal access agreements," 688

Masters, Dehorah C., appt., 727

Matthews, Judith, “NSF MetaCenter Computational Science Highlights $^{n}$ \& logo, 575

Matzek, Richard A, news note, 109

Mayoh, Helen, retired, 203

Mech, Terrence F. "Becoming a leader on campus," 409-12

"Meet the candidates for ACRL president," 772

"Meet the editors," ACRL, 333

Meissner, Arolana, news note, 109

"Memorial resolution honoring William Andrew Moffett (1933 1995)," 232

Menendez, Miguel M., appt., 727

Merriman, Faith, "Using the Internet in serials management," $148-50,176$

Metter, Ellen, "Madonna in the hallowed halls," 710-11

Metz, Ray E., photos., 560, 808

Meyer, Richard, "EDUCOM '94: the promise of technology," 22-23; "Focusing library vision on educational outcomes," 335-37, comment, 466

Miami Book Fair International, 1995, Scharf, poster (photo. cover, no. 10)

"Michigan library gets facelift" \& photo., 534

Miller, Arthur H., Jr, news note \& port., 427

Miller, James P., retired, 667

Miller, William, ACRL Vice-President \& port., 483-84; photo. 561; "Plans for ACRL," 181-83 (port., 178)

Millson-Martula, Christopher, appt.. 785

Miriam Dudley Bibliographic Instruction Librarian Award $1995,258,260 ; 1996,563-64$

"Mr. Peacock" photo., 749

Moffett, William A "Colleagues and friends honor Bill Moffett" \& port. 383; cleceased \& port., 286-87; "Memorial resolution." 232

Mollema, Peter C., Jr, deceased, 584

Montavon, Victoria, photos., 560-61, 808

"More on information literacy data" 231, 382

Morgan, Keith, "Association of Research Libraries (ARL) Statistics," 494-95; "United States Department of the Treasury," 190-91

Morton, Bruce, appt., 582

Mosher, Paul, news note, 726

Mountainside Pub. Co., Miriam Dudley Biblingraphic Instruction Librarian Award, $1995,258,260 ; 1996,563-64$

Mudrock, Theresa, photo., 264

MUSE Educational Media, "Getty pilot project explores use of digital images," 139

MLA, Walter Gerboth Award, 1995,426

$\mathbf{N}$

"Nashville Cuh riverboat," ACRL, National Conference, 1997, photo., 377 
"NASA Spacelink," Wagner, 420-21

NCES, "Coming in '96: IPEDS adds new data items," 622; "Getting the 1992 library data," 8

National Forest Dude Rancb Vacations, directory (cover, no. 6)

NLW, "Apply for the NLW grant," 76; "A time to celebrate" (logos \& posters), 76-79

"The National Museum of American Art," Dodd, 714-15

"NSF MetaCenter Computational Science Highlights" \& logo, Matthews, 575

National Women's History Project, "Celebrate Women's History Month" \& posier, 138; "Celebrating Black women's history," poster available, 72

Neal, James G., appt. \& port., 503-4

Nekritz, Leah K, rerired \& port., 583

"NetVet" \& logo, Lee, 420-21

Neumann, Joan, retired, 47

"New book banishes stereotypes," ACRL, 73

"New Grainger Library at Univ. of [1]." \& photo., U. of Ill., UC, $7-8$

New Mexico State U. "Teaching with scratch paper," 160,183

"New MLA listserv," ACRL, 623

"New national numbers on academic libraries," Lynch (55:63031), data available, 8 , updates, $75,231,382$

"New publications," Eberhart, 35-37, 105-6, 193-94, 279-80 ( 8 photo.), 348-49, 422-23, 497-99 (\& photo.), 576-77, 661, $663,718-21,781-82$ (\& photo)

"News from the field," Davis, 5-8, 71-75, 137-40, 231-34, 311$13,379-83,451-54,531-34,619-23$ ( 8 s photo.), 687-90, $751-54$

"The Nine Planets: a multimedia tour of the solar system," Nolan, 347

Nitecki, Danuta A., "Doctoral Dissertation Fellowship awarded" \& port., 260; photo., 569

Nolan, Christopher W. "The Nine Planets: a multimedia tour of the solar system," 347

"NATO gopher," Stemmer, 574

Northern Pacific Railway Co., National Forest Dude Rancb Vacations, directory (cover, no. 6)

Northwestem U., Africa seen by American Negroes, map (cover, no. 2)

"Nurseryman's plates," U. of Rochester, 440

Nutter, Susan K., appt. \& port., 785-86

O'Neal, Sally, retired, 583

Oberlin Coll., "Colleagues and friends honor Bill Moffett" \& port., 383

Oberly (Eunice Rockwell) Award, 1995,262

"Ohio University celebrates two million volumes," 754

"OhioLINK marks fifth year," 138

"OhioLINK online horrowing system increases book requests ten times," 752-54

Oliver, Leslie Mahin, deceased, 729

"Online Mendelian Inheritance in Man," Blackmer, 421

"Online resources for Internet trainers," Weissinger, 535-39. 572

"Only Post-It on expendable items," 623

Oram, Richard W., "Wins award for best article in RBML" \& port., 265

"Order your Annual Conference audiocassettes," 553

"Oregon libraries create Orbis," 619

Osif, Bonnie, news note \& port., 581

"Overdue notices: cutting costs and saving time," Horn, 8

\section{$\mathbf{P}, \mathbf{Q}$}

Pamplin, Elma Cole, deceased, 287

Pao, Miranda Hsu-Yung, deceased, 668

Papers solicited, 398

Pappas, David L. appt., 665

"Paris Pages: a collection of everything regarding the City of light" \& logo, Larsen, 659-60
Parker, Diane C., "Standards for college libraries': founda tions," 330-31, 337

Parrish, Jerral R., deceased, 729

Parsons, A. Chapman, deceased, 113

Parsons, Gerald E, deceased, 584

"Paula," Stieglitz (photo., cover, no. 8)

Paulk, Janet, retired, 286

Peck, Steven Dudley, deceased, 47

"People in the news," Spiegel, 43-47, 109-13, 202-3, 283-87, $352-54,426-29,503-5,664-68,726-29$, 785-87; Thompson, 581-84

Petty, Jenny, letter to the ed., 645

Pfeiffer, Carol, photo., 561

Phillips, Linda, photos., 560, 808

Piette, Mary I., news note, 664

Pinto, David, appt., 786

"Pittsburgh a success!" ACRL, National Conference, 1995, \& photo., 311

"Pittsburgh: a walk around town" \& photo., Lowry, 95-97

"Plans for ACRL," Hahn \& Miller, 178-83

Poland, Jean A., photo., 729

"Pooled endowments: a new funding idea," Basefsky, 405-7

Pope, Nolan F., news note, 664

PCA, "Call for popular culure papers," 398

Potter, Cynthia, appt., 665

Potter, William Gray, news note, 43

"Preliminary program," ACRL, National conference, 1995, P 1--P-20 (no. 1)

"Preservation news," Hedberg, 28, 100, 188, 275, 345, 419 $491,573,658,713$

"President Clinton dedicates new UConn research center" \& photo., 751-52

Program for Art on Film, "Moves to Columbia," 140

"Purdue links Web to federal documents," Purdue U., 451

"Racial \& ethnic diversiry: information exchange," Absion, 31 276, 716-17

Rader, Hannelore B., "EBSS Award" \& port., 261

Radford, Marie \& Gary, phoro., 476

Rare Books and Manuscripts Librarianship Award, 1995, 265 1996,569

Ratliff, Neil, deceased, 354

Raymond, Lorraine, retired, 583

"Readmore sponsors Backserv," 381

Ready, Sandra, photos., 560, 808

Regner, Erlinda J., news note, 202

Reichel, Mary, photos., 560-61, 808

Renaud, Robert, "Building the digital library," 547-48; "Choos ing our futures: ACRL's 8th National Conference," 394-95

"Research across the curriculum," McKinzie, 414, 417, comment, 645

"Research agenda for college librarianship," ACRL, 470-71, 485, comments, 645

RLG, "Russian state archives will be added to RLIN," 71, 73

"RLG initiates new record service with Casalini Libri," 754

Retirements, 47, 112, 203, 285-86, 429, 505, 583-84, 667-68 787

Rettig, Jim, "'Rettig on Reference' debuts on the Internet, " 688 89

"Rettiz on Reference' debuts on the Internet," $688-89$

"Revision of the mission of a university undergraduate library model statement," ACRL, 339

Rhodes, Diane B., "Oberly Award" \& port., 262

Rible, Jim, "The 7.001 Hypertextbook," 493, logo, 494

"Rice replaces NOTIS with UNICORN," 619

Richards, Berry G., retired \& port., 584

Richards, Susan, appt. \& port., 727

Richardson, John V., Jr., news note, 664

Ridgeway, Trish, news note, 43

Riggs, Donald E., "Named College Er research libraries editor" $\&$ port., 380 
Roark Derrie E., "Community College Award" \& port., 26465

Robare, Lori, "The Human-Languages Page," 660

Robinson, Judith G., appt., 110, 427

Rockman, Ilene F., "Coping with library incidents," 456-57

Rockwood, Irving E., duties \& port., 649; "Named editor and publisher of Choicen \& port, 453

Rogers, Sharon J., news note, 43

Rossi, Gary, deceased, 429

Runkle, Martin, news note, 726

Russian State Archival Service, "Kussian state archives will be added to RLIN," 71,73

"Russian state archives will be added to RLIN," RLG, 71, 73

Ryan, Terry, news note, 43

\section{$S$}

"St. Petershurg Jr. College reorganizes for the ' $90 \mathrm{~s}_{1}{ }^{2}$ Anderson, 541-46

Samuel Lazerow Fellowship, 1995, 260-61; 1996, 568

Sander, Richard, photo, 233

Sassé, Margo, appi., 427

Sassen, Saskia, photo., 387

Saunders, Laverna M., appt. \& port., 45

Saur (K. G.) Award for Best College \& Research Libraries Article, 1995, 260; 1996, 569

Scalf, Katie, photo., 312

Scharf, Kenny, Miami Book Fair International, 1995, poster (photo., cover, no. 10)

Scherdin, Mary Jane, Discovering librarians: profiles of a profession, published, 73

Schmidt, Karen A., "Receives Lazerow Fellowship" \& port., 260-61

"Scholarly Societies Project," Luck, 493-94

Schreiner, Lyle R., retired \& port., 47

Schrodt, Paul, appt., 202

Schwartz, Charles A., "Receives K. G. Saur Award" \& port., 260

Science and Engineering Conference proceedings: a guide to sources foridentification and verification $\&$ photo., ACRL, 453-54

Scott, Edward A., appt., 665

Scott, R. Neil, letter to the ed. \& response, 645

Scotr, Willie, retired, 203

"Search committees aid ACRL," 451, 453

Searcy, Herbert L., deceased, 584

Seetoo, Amy D., news note $\&$ porr., 202

Seidman, Ruth K., news note \& port., 283

"Selecting electronic journals," Grochmal, 632-33, 654

"Selection criteria for Internet resources, ${ }^{"}$ Cassel, $92-93$

Seng, Mary, retired, 584

"The 7.001 Hypertextbook, "Rible, 493, logo, 494

Shapiro, Beth Janet, deceased \& port., 668

"Share your library's news," 47,402

"Share your opinion with CERL news readers," 400

Shepard, Martha, retired, 203

Shipman, John S., retired, 584

Shoujing, Zhuang, news note, 581

Siggins, Jack A., appt, 110

Singerman, Robert, news note, 43

Slonaker, Ethel Houtz, deceased, 505

"Slovenija," Valentine, 103

Small, John, "Baltics Online," 190

Smith, Amy Sherman, "ALADN: a new network for fundraising," 329; "Library fundraising tips and resources," 328

Smith, Elizabeth, "Facing the challenge of democratization," 324-25

Smith, Linnie U., "Hosting future librarians" \& photo., 481-82 Smith, Robert, port., 351

Smithsonian Institution Libraries, "Available on Internet, " 233

"Smithsonian Libraries available on Internet," 233

Snelson, Pam, photo., 476

"SAA's Preservation Management Training Program is a huge success, "Walters, 139
Somerville, Mary R., "Candidates for ALA president" \& port., 267.68

Souter, Thomas A., retired, 667-68

Southern IIl. U., Carbondale, "Distance learning classroom opened," 5

Spain, Victoria J., "Internet resources for education," 153-57, correction, 320

Spalding, Helen, "Financial report" \& port., 809-813; photos. 560-61, 808

Speller, Benjamin, pon, 351

Spiegel, Pam, "ACRL honors the 1995 award winners," 258-65: duties \& port., 649; "People in the news," 43-47, 109-13, 202-3, 283-87, 352-54, 426-29, 503-5, 664-68, 726-29, 785 87; rev. (The whole library bandbook 2), 577; "Rockwood named editor and publisher of Cboice" \& port., 453

Stam, David, news note, 726

"Standards for college libraries," ACRL, 1995, approved, 245 57 , foundations, 330-31, 337

"'Standards for college libraries': foundations," Parker, 330-31, 337

"Stanford creating digital Dewey for Internet, , Stanford U., 231

SUNY, Albany, "Voyage window" photo, 6

"SUNY-Albany bookmarks available," 6

Steele, Victoria, news notes \& ports., 283, 664

Stemmer, John K., "NATO gopher," 574

Stephen, Ross, retired, 787

Sterling, Michael, duties \& port., 649

Stieglitz, Alfred, "Paula" (photo., cover, no. 8)

Stoffle, Carla, "Applause for ACRL's support of ALA Goal $2000, " 270$

"Strategic Planning Committee," ACRL, 401

Striman, Brian, "Internet resources for law," 18-20

"STS discussions at Midwinter," ACRL, 8

"STS seeks new logo," 234

"Student retention and academic libraries" \& photo., Kelly, $757-59$

Stussy, Susan A., appt., 582

Sullivan, Peggy, appt. \& port., 727

Swank, Raynard Coe, deceased, 505

Sweeny, Mary Kay, retired, 47

Switzer, Teri R., "Ergonomics: an ounce of prevention" \& drawing, 314-17

T

Tabb, Bruce H., photo., 729

Takaki, Ronald, photo., 386

Taylor, Cynthia, duties \& port., 650

"Teaching, léarning, and technology roundtable," AAHE, National Conference, 1995, Hoffman, 326, 344

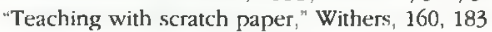

"Tell them you saw it in CERL neus," 458

Tenglund, Ann M., "Financial Aid Information Page," 778 (\& logo, 779)

Tex. A\&M U. "Center explores digital libraries' potential," 379

"Thanks for your support," ACRI, 467

"There's still time to bid on two silent auction items," ALA, 708

Thompson, Hugh, duties \& port., 650; "Grants and acquisitions," 39-40, 107-8, 198-200, 281-82, 350-51, 424-25, 500$501,578-79,662-63,723-25,783-84$; "People in the news," 581-84

Thompson-Wise, Deborah, news note, 503

"A time to celebrate," Wallace (logos \& posters), 76-79

"The time to lead," ACRL, 270

"Time to put the Internet in perspective," Jacobsen, 144-47

Tompkins, Philip, appt \& port., 665

Topper, Elisa F., duties \& port., 650; "Joins ACRL staff," 61923

"Transborder ILL guidelines available from ARL" 687

Trenton State Coll., "Helping students delve deeper into books, ${ }^{, 7} 704-5$

"Tribal Voice/PowWow," Wagner, 715

Troll, Denise A., "What's hot and what's not," 236-39 
"Truant selling Saturday Evening Post, St. Louis, Mo., 1910," Hine, photo., 529

Trump, Alfred G., deceased, 113

U

"ULS husy at ALA in Chicago," 534

"ULS undaunted by wintry Philadelphia," Wallbridge, 242-43

"UnCover Reveal adds features," 379

"United Nations gopher, ${ }^{n}$ Malone, 277

"United States Department of the Treasury," Morgan, 190-91

"Univ, of Alberta outsources cataloging," 140

UC, Berkeley, "Welcome freshmen with summer reading," 451

"UCSD library renamed to honor Dr. Seuss" \& photo., 690

U. of Chicago, "The merchandise mart" photo., 309; "Waiting," drawing (cover, no. 5)

"Universiry of Cincinnati uses cable TV" \& phoro., 311-12

U. of Colo., "Applying for professional positions," 415-17; "Chinook blows into CU-Boulder libraries," 233

U. of Conn., Storrs, "President Clinton dedicates new UConn research center" \& photo., 751-52

U. of 1ll., Chicago, "Student retention and academic libraries" \& photo., 757-59

U. of Ill., U-C, "New Grainger Library at Univ, of 1ll." \& photo., 7.8

U. of Md., "Paula" (photo., cover, no. 8); "Truant selling Sanurday Evening Post, St. Louis, Mo., 1910" photo., 529

U. of Mich., "Africana file available," 73; "Journal of electronic publishing created," 71: "Library gets facelift" \& photo., 534

"University of Michigan reinvents library education," 138

U. of Okla., "Overdue notices: cutting costs and saving time,"

"University of Oregon expands library" \& photo., 73-74

U. of Rochester, "Clematis Jackmanii" (photo., cover, no. 7); "Nurseryman's plates," 449

U. of Southem Calif., "Everything you ever wanted to know ahout L.A.," 379

"USC chooses Ameritech's Horizon," 619

U. of Tex., Austin, "Fashion illustration" (photo., cover, no. 11); "Mr. Peacock" photo., 749

U. of Vt., "Benefit book auction a success," 5

U. of Wash., "Project wins BIS Innovation Award" \& photo, 264

U. of Wisc.-Milwaukee, Middle East Map Collection photo, 685

"Update on information literacy data," ACRL, 75, updates, 231, 382

"Using the Internet in serials management," Kascus, 148-50 176

v

Valauskas, Edward J., photo., 729

Valentine, Barbara, "Slovenija," 103

veldof, Jerilyn, "ACRL in Nashville: a focus on dialogue," 646

Vierra, Tricia, photo., 729

Va. Commonwealth U., "Hosting future librarians" \& photo. 481-82

"VCU game benefits Black History Archives Project" \& photo. 233-34

"Virginia creating virtual library," 6-7, 313

$\mathrm{Va}$. Tech., "Building preservation awareness" \& posters, 707

"Virtual lihrary of Virginia adds Ariel," 313 (creation, 6-7)

"Visit the ALA homepage," 534

Vogel, Jane, retired, 112

"Voice of America gopher," Horne, 32-33

Vondracek, Rurh, "Classics and Mediterranean archaeology," 32

Vormelker, Rose L., deceased, 113

"Vote Smart Web (Project Vote Smart)," Hupp, 714 (logo, 715)

"Voyage window" photo., SUNY, Albany, 6
$\mathrm{W}, \mathrm{x}$

Wagner, Kurt W., "NASA Spacelink," 420-21; "Tribal Voicet PowWow, 715

"Waiting," Longstreet, drawing (cover, no. 5)

Walbridge, Sharon, "ULS busy at ALA in Chicago," 534

Walker, Paula, photo., 264

Walker, Rosemary E., appt., 353

Walker, William, news note, 43

Wallace, Linda K., "A time to celebrate" (logos \& posters), 76 79

Wallbridge, Sharon, "ULS undaunted by wintry Philadelphia," 242-43

Walter Gerboth Award, 1995, 426

Walters, John Spencer, news note, 581

Walters, Tyler O., "SAA's Preservation Management Training Program is a huge success," 139

Wang, Andrew H., news note, 202

"Washington hotline," Bradley, 98-99, 186-87, 274, 343-44, $418,488-89,657,712,725$

Waugh, Kappa, cartoons, 534, 689

The way I see it ("Customer service? not really," Hoadley, 17576; "Focusing library vision on educational outcomes," Meyer, 335-37, comment, 466; "Madonna in the hallowed halls," Metter, 710-11; "Research across the curriculum," McKinzie, 414, 417, comment, 645)

Weaver-Meyers, Pat, "Overdue notices: cutting costs and saving time," 8

"Web Day in Portland, Oregon," Klein, 688

"Wedding doesn't stop conference attendance" \& photo., 53334

Weissinger, Nancy 3., "Online resources for Internet trainers," $535-39,572$

"Welcome freshmen with summer reading," UC, Berkeley, 451

Wells, Ellen Baker, deceased, 429

Werking, Richard Hume, "The ACRL publications program" \& phoros., 332-33

West, Sharon, appt, 110

Whaley, John, photo., 233

"What's hot and what's not," Troll, 236-39

"What's new? An update from Pittsburgh" \& photos, ACRL, National Conference, 1995, 385-92, correction, 548

"What's new, part two: more from Pittsburgh" \& photos. ACRL, National Conference, 1995, 473-79

Whelan, Mary H., retired, 505

"Who uses the Internet?" 689

The whole library handbook 2: current data, professional advice, and curiosa about libraries and library services, Eberhart, comp., rev. of, 577

Wildman, Iris, retired, 429

Wilson, Lizabeth (Bersy), "Named Dudley Bibliographic Instruction Librarian" \& port., 258, 260

Winherry, Carol, retired, 286

Withers, Carol, "Teaching with scratch paper," 160, 183

Wold, Shelley T., retired, 505

"Working with faculty to build collections," Jenkins, 322

Wyatt, Roger, news note, 43

$Y$

Yang, Eveline, photo., 135

Yavarkovsky, Jerome, appt., 665-66

Yelich, Nolan T., appt., 427

Z

Zaclner, Par, retired, 787

Zald, Anne, photo., 264

Zaporazhetz, Laurene E., news note, 352

Zemon, Mickey, response to letter to the ed., 645

Zia, Dora, retired, 47

Zink, Steven D., appt., 427

Zula, Floyd, letter to the ed. \& response, 780 


\section{Introducing \\ The Essential References of Dur Time}

\section{Visit the AP Booth at ALA}

\section{Encyclopedia of Virology Plus} Ediled by

R.G. Websler and

A. Granoti

Stand-Alone: $\$ 475.00$

danuary 1996/1SBN. 0-12-000103-9

For mone information regyding a network

version, please contact the Publisher.

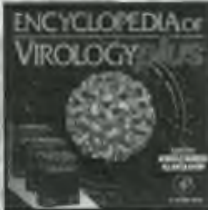

Base price allows for up to five users!

Methods in Enzymology

on CD-ROM, CD-1

Recombinant DNA and

Related Methodology

18 Selested Core Volumes with

Cumulative Indexes for MIE

Volumes 1-244

Includes Recombinant

DNA, Parts A-1, DNA

Structure, Parts A \& B,

Three Guide to's, and

Related Volumes

sories Eoriors-in-Chiel

John N. Abelson and Melvin I. Simon

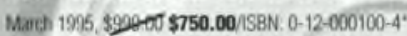

- Price reduction valid through March 31, 1996

\section{Methods in Enzymology}

Index, CD-ROM 1

December $1995,399.00 /$ ISBN: 0-12-000101-2

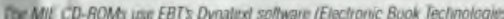
inc. Arovidence Rhode lsand). and were developed oy Lightinders of San

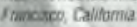

Order from your local bookseller or directly from

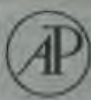

Academic Press, Inc.

Order Fulfillment Dept. OM27103

6277 Sea Harbor Dives. Orlando. FL. 3288

2428 Oval Pasd. London NWI 7DX UK

in the US and Canoda

CAII TOL FREE: 1 . 0000215064

ENC $1900-995.7377$

Emat apeacad.com

In Europs CALL 0181-300-3322

Visit os on the Worid Wide Weo - htte//nww apnet.com/

Prices satijd b ctungos withoul nobses 01906 by Acodomic Pruss, inc Al Plogite Pesenved.
The Academic Press Dictionary of Science and Technology was recognized as one of American Libraries' Outstanding Relerences of 19931

Academic Press Dictionary of Science and Technology CD-ROM

VERSION 1.0

Novemite 1995. $\$ 79.96$

ISBN: 0-12-200401-9

A discount of $75 \%$ oft the print version is availabie DIRECTLY FROM

ACAOEMIC PRESS whin you buy

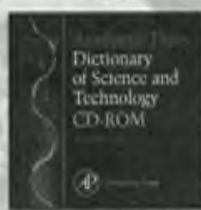

the CO-ROM-us quote ISBN $0-12-200399-31$

Encyclopedia of Physical Science and Technology, Second Edition, CD-ROM

VERSION 1,0

Edited by

Robert A. Meyers

Introductory offer: $\$ 2395.00^{\circ}$

December $1995, \$ 2995.00$

ISBN 0-12-000200-0

-Eflective through Maich 31, 1996.

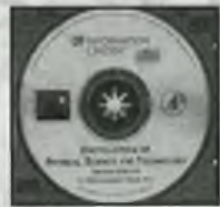

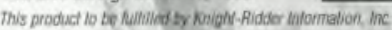

\section{KR INFORMATION} ONDISC

The Nucleus of Knowledge

Encyclopedia of

Analytical Science

Edilor-in-Chiet

Alan Townshend

Ten-Volume Set

June 1995, 6059 DD .\$1950 00/198N 0-12-2267u0-1

\section{Encyclopedia of} Environmental Biology

Edited by

William A. Nierenberg

Three-Volume Set

June 1995, 2114 pp., \$475.00/ASBN: 0-12-226730-3 
ing copyright owners' effective control over data resources and stifle innovation and job creation in the private sector with overbroad prohibitions against manufacture and sale of legitimately useful consumer electronic devices. ALA continues to argue that to reduce educators' and the public's access to digital information by creating a new "transmission right" would make electronic communications "distributions" within the meaning of the Copyright Act. The White Paper would categorize even "browsing" as a potentially infringing "reproduction." (For additional information about the DFC, its other members, and activities contact Adam M. Eisgrau at the ALA Washington Office at (202) 628-8410 or e-mail: AME@alawash.org.)

\section{Copyright term extension legislation}

Legislation to extend the term of copyright from "life-plus-50" to "life-plus-70" years remains pending before the Judiciary Committees of both Houses of Congress. Consideration of the measure, however, remains stalled while negotiations on another bill (regarding the licensing of music by commercial and other establishments) continue. Al.A, acting in concert with four other national library organizations, has written to the chairmen of both committees requesting that the legislation be amended to provide libraries with the authority to "use" copyrighted works during the 20-year extension period. Language crafted by the library groups, and endorsed by Register of Copyright Marybeth Peters, would create the presumption that such use was permissible provided that it was neither undertaken with a prior profit motive, nor subsequently affected the market for the copyrighted work adversely. At ALA's request, House Courts \& Intellectual Property Subcommittee chairman Carlos Moorhead (RCalif.) wrote to Peters on November 29 requesting that she bring library and copyright owner industry representatives together to negotiate the text of a "library" amendment acceptable to both groups. At this writing it appeared likely that this meeting, perhaps the first of several, would take place between December 11 and 22. Lilbrary groups are acting under the assumption that time is of the essence. While no meeting of Moorhead's subcommittee to consider the term extension bill has been scheduled, should the music licensing issue be resolved, such a session could rapidly be convened before Congress' holiday recess.

\section{(Bill cont. from page 33)}

access to information on the grounds that it is perceived to be frivolous or lacking value," wave red flags before administrators of campuses. Moreover, just because a professor wants extensive materials in a controversial area, for example, he/she should not warrant ALA Intellectual Freedom championing any more than the professor with a similar level of unrealistic acquisitions expectations in a narrow area of the Classics.

3.) Since Annual Conference another related concern has arisen. It comes from a response made by Judith Krug, who is the very heart and voice of Intellectual Freedom, to a question asking the relationship between intellectual freedom and intellectual participation. In the September 1995 American Libraries, she is quoted as saying that "they're one and the same." We do not believe that these issues are the same or that intellectual participation is "just the next step after intellectual freedom."

In fact, the major information problem - the intellectual participation problem-confronting most people today is not having access to or not knowing what information is available to help them address the problems of their daily lives. These information needs can and do run the gamut from how I can apply for unemployment, to where I can find reliable affordable child care, to what financial help I can get to go to college, to information on a recently diagnosed illness. And ALA's efforts within its Goal 2000 to address this larger issue should not be diminished to an add-on of the IFC's agenda.

As to the electronic interpretation, ACRL, is again forwarding to you the memo it gave to you at the annual conference which raises specific concerns that it has regarding the 1.4 version. It would be our hope that the IFC would seriously consider these concerns as well as those raised by other people and make changes so as to allow us to support the interpretation. We also hope that the IFC will give substantive attention to the larger issues raised in this memo and will seek to work more cooperatively with ALA divisional leadership. Meanwhile members of our Board will work with ACRL's IFC in developing specific wording changes in the electronic interpretation for what it will forward to your committee prior to Midwinter. 